\title{
Propiedades psicométricas de instrumentos de evaluación de aspectos psicosociales en adolescentes de México
}

\author{
Psychometric Properties of Instruments for Assessing \\ Psychosocial Aspects in Adolescents from México
}

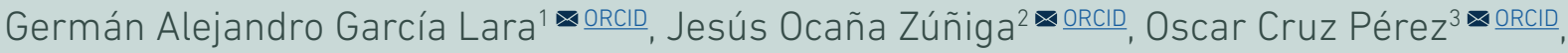 \\ Soledad Hernández Solís ${ }^{4} \underline{\mathrm{ORCID}}$, Carlos Eduardo Pérez Jiménez ${ }^{5} \underline{\mathrm{ORCID}}$
}

Universidad de Ciencias y Artes de Chiapas - UNICACH-

México

Fecha correspondencia:

Recibido: septiembre 19 de 2018.

Aceptado: octubre 15 de 2019.

Forma de citar:

García Lara, G. A., Ocaña Zúñiga,

J., Cruz Pérez, O., Hernández Solís,

S., \& Pérez Jiménez, C.E. (2020).

Propiedades psicométricas de

instrumentos de evaluación

de aspectos psicosociales en

adolescentes de México. Rev. CES

Psico, 13(1), 70-88.

Open access

(c) Copyright

Licencia creative commons

Ética de publicaciones

Revisión por pares

Gestión por Open Journal System

DOl: http://dx.doi.org/10.21615/

cesp.13.1.5

ISSN: 2011-3080

Sobre los autores:

1. Doctor en Educación. Profesor investigador, integrante del Cuerpo Académico: Educación y Procesos

Comparte

\section{Resumen}

El estudio tuvo como objetivo analizar las propiedades psicométricas de las escalas de Ideación Suicida de Roberts, de Depresión del Centro de Estudios Epidemiológicos [CES-D] de Radloff, de Autoestima de Rosenberg, y de Impulsividad, Relación con Papá y Relación con Mamá, las tres de Climent, Aragón y Plutchick. Se realizó un estudio no experimental, de tipo transversal ex-post facto con 4,759 adolescentes de ambos sexos del estado de Chiapas, México. La confiabilidad evaluada a través del coeficiente alpha de Cronbach reportó índices superiores a .700 para todos los instrumentos, excepto para la Escala de Impulsividad. Los análisis factoriales exploratorios realizados mostraron arreglos de ítems semejantes, más no iguales, a los reportados en otros estudios en los que se han aplicado estos instrumentos, confirmando la validez de los constructos teóricos en los que se basó su diseño. Las Escalas analizadas son válidas para su uso en adolescentes de México, considerando los puntos de corte establecidos para cada una de ellas.

Palabras clave: Propiedades Psicométricas, Adolescentes, Escala de Ideación Suicida, Escala de Depresión CES-D, Escala de Autoestima, Escala de Impulsividad, Escala de Relación con Papá, Escala de Relación con Mamá.

\section{Abstract}

The study aims to analyze the psychometric properties of Roberts' Suicidal Ideation Scale, Radloff' Center for Epidemiologic Studies Depression Scale [CES-D], Radloff, Rosenberg Self-Esteem, and Impulsiveness, Dad Relationship and Mom Relationship, the three of Climent, Aragón \& Plutchick. A non-experimental, cross-sectional, ex post facto study was performed with 4,759 adolescents of both sexes in Chiapas, Mexico. Reliability through Cronbach's alpha coefficient reported higher rates to .700 for all instruments, except for Impulsiveness Scale. The exploratory factor analyzes showed items such arrangements, but not identical, to those reported in other studies where these instruments have been administered, confirming the validity of the theoretical constructs on which its design was based. The scales are valid for use in adolescents from México, considering the cut-off points established for each of these tools. 
Sociales Contemporáneos de la Facultad de Ciencias Humanas y Sociales de la UNICACH.

\section{Doctor en Ciencias en} Desarrollo Sustentable. Profesor investigador, integrante del Cuerpo Académico: Educación y Procesos Sociales Contemporáneos de la Facultad de Ciencias Humanas y Sociales de la UNICACH.

3. Doctor en Ciencias Sociales. Profesor investigador, líder del Cuerpo Académico: Educación y Procesos Sociales Contemporáneos de la Facultad de Ciencias Humanas y Sociales de la UNICACH.

4. Doctora en Educación. Colaboradora del Cuerpo Académico: Educación y Procesos Sociales Contemporáneos de la Facultad de Ciencias Humanas y Sociales de la UNICACH.

5. Maestro en Teoría Crítica. Colaborador del Cuerpo Académico: Educación y Procesos Sociales Contemporáneos de la Facultad de Ciencias Humanas y Sociales de la UNICACH.
Keywords: Psychometric Properties, Adolescents, Suicidal Ideation Scale, Depression Scale CES-D, Self-Esteem Scale, Impulsiveness Scale, Dad Relationship Scale, Mom Relationship Scale.

\section{Introducción}

La población adolescente constituye un grupo social altamente vulnerable ante las experiencias contradictorias y ambivalentes que se generan en el proceso de individuación y construcción de la identidad, lo que disminuye su capacidad para afrontar distintas problemáticas psicosociales como la ideación suicida, sintomatología depresiva e impulsividad, triada estrechamente relacionada entre sí (Auerbach, Stewart, \& Johnson, 2017; Bi et al., 2017; Silva et al., 2017; Sarkisian, Van Hulle, \& Goldsmith, 2018); a lo que en ocasiones se suma la baja autoestima, resultado de la interacción dinámica de diversos acontecimientos históricos y del desarrollo, no como una condición interna, sino como una organización funcional que deriva de la interacción con otros (Sánchez. Villarreal, \& Musitu, 2013); dificultades en las relaciones familiares (Silva et al., 2017; Estévez, Jiménez, \& Moreno, 2018); uso y abuso de sustancias psicoactivas, y conductas sexuales de riesgo, entre otros aspectos (Caballero, Suárez, \& Bruges, 2015; Palacios, 2015).

En México, 3.4\% de adolescentes de entre 12 y 17 años presentan ideación suicida, en una proporción de 3-1 para mujeres y hombres, respectivamente (Borges et al., 2019); la sintomatología depresiva registra una prevalencia en un rango entre $10 \%$ y $16 \%$, siendo mayor en mujeres (González, Hermosillo, Vacio, Peralta, \& Wagner, 2015); el consumo de drogas ilegales en el último año fue de 3.1\% (INPRFM, 2017a); y, poco más de $70 \%$ utiliza condón en su primera relación, porcentaje que disminuye en relaciones subsecuentes (Encuesta Nacional de Salud y Nutrición [ENSANUT 2012], en Gutiérrez et al., 2012), lo que aumenta las posibilidades de un embarazo a esta edad. De manera particular, en el estado de Chiapas, en adolescentes con edad promedio de 18 años, se reportó que 7.3\% presenta ideación suicida, 13.3\% sintomatología depresiva y 5,5\% impulsividad (García et al., 2018); en escolares de 12 a 17 años, 1.2\% consumió drogas ilegales en el último año (INPRFM, 2017b), y en adolescentes de 18 años, $81 \%$ utilizó condón en su primera relación sexual (García, Ocaña, Hernández. \& Cruz, s/a). Como se aprecia, el consumo de drogas alcanza porcentajes menores en la entidad chiapaneca, en tanto que el porcentaje de casos con sintomatología depresiva, impulsividad o el uso de condones, es similar a lo reportado en el país. La ideación suicida presenta porcentajes mayores a la media nacional, probablemente por la edad de la muestra, pues de acuerdo con la OMS (2019) este comportamiento se presenta en mayor proporción en personas de 18 a 39 años (OPS, 2014).

Tales problemáticas pueden afectar a los adolescentes en ámbitos como el académico y las relaciones psicosociales (Silva et al., 2017; Estévez et al., 2018); o bien, mantenerse y perdurar aún en etapas posteriores de desarrollo. Su impacto en la pérdida de años de vida saludable, la elevada tasa de morbilidad que le acompaña y una fuerte demanda al sistema sanitario, es de suma relevancia para la salud pública (Berenzon et al., 2013; González-Forteza et al., 2011); no obstante, su medición en grupos poblacionales de alto riesgo como la población adolescente, no siempre resulta factible ante la falta de pruebas de tamizaje para la detección oportuna de casos de riesgo.

Existen diversos instrumentos que evalúan variables como la sintomatología depresiva y la ideación suicida en muestra clínicas, por ejemplo, el Patient Health Questionnaire (Baader et al., 2012) o el Cuestionario de Riesgo Suicida (Horowitz et al., 2012), no así 
Pág 72

Para el estudio de la sintomatología depresiva, considerada una de las afectaciones psíquicas más frecuentes y la principal causa de discapacidad en la sociedad contemporánea (Tomitaka et al., 2018), uno de los instrumentos más utilizados es la Escala de Depresión del Centro de Estudios Epidemiológicos [CES-D] (Radloff, 1977), validada en investigaciones de reciente publicación (Baron, Davies, \& Lund, 2017; Franco-Díaz, Fernández-Niño, \& Astudillo-García, 2018). en población general; contexto en el que también es necesario poseer herramientas confiables, válidas y sensibles para la detección de casos con dichas problemáticas.

Para el estudio de la sintomatología depresiva, considerada una de las afectaciones psíquicas más frecuentes y la principal causa de discapacidad en la sociedad contemporánea (Tomitaka et al., 2018), uno de los instrumentos más utilizados es la Escala de Depresión del Centro de Estudios Epidemiológicos [CES-D] (Radloff, 1977), validada en investigaciones de reciente publicación (Baron, Davies, \& Lund, 2017; Franco-Díaz, Fernández-Niño, \& Astudillo-García, 2018). Así, en su versión de 20 ítems, se aplicó a 518 adultos austriacos de 18 a 65 años y se obtuvo una estructura de cuatro factores: afecto positivo, afecto negativo, síntomas somáticos y dificultades interpersonales. Los autores señalan que el factor dificultades interpersonales arrojó bajos niveles de confiabilidad (Alexandrowicz, Jahn, \& Wancata, 2018); también se aplicó a 600 adultos indígenas de Estados Unidos (Gray, Brionez, Petros, \& Gonzaga, 2018) probándose su validez y confiabilidad; en su versión de 8 reactivos fue administrada en adultos de 13 países de Europa, detectando un mayor número de casos con síntomas depresivos que con la Escala Euro-D (Courtin, Knapp, Grundy, \& Avendaño, 2015); en su conformación de 10 reactivos se ha aplicado en población indígena estadounidense, adolescentes y jóvenes adultos, obteniéndose una solución de dos factores, no consistente con datos conseguidos en otros grupos indígenas, lo que indica, en este caso, la necesidad de considerar las características socioculturales de la muestra (Harry \& Crea, 2018); se utilizó también en un estudio realizado con 5,434 adolescentes chinos en el que la Escala presentó una estructura de un solo factor y se señaló que sus propiedades psicométricas son adecuadas para emplearla como instrumento de tamizaje (Yang et al., 2018); y en minorías sexuales, jóvenes y adultos de diferentes países de Asia Pacífico, donde también se obtuvo una estructura de un solo factor, útil para comparar sintomatología depresiva entre minorías sexuales y no sexuales de esta región (Miedema, Haardörfer, \& Yount, 2018); en su versión de 16 reactivos se administró a adultos irlandeses, cuyos resultados indican que los reactivos siguen un mismo patrón de respuesta entre los sujetos (Tomitaka et al., 2018).

En México, desde hace más de tres décadas se han realizado estudios para analizar las propiedades psicométricas de validez y confiabilidad del CES-D (Masten, Caldwell-Colbert, Alcalá, \& Mijares, 1986). Uno de los primeros trabajos que exploran su estructura factorial en adolescentes es el de Mariño, Medina-Mora, Chaparro y González-Forteza (1993, citados por González-Forteza et al., 2011), quienes reportan tres factores: afecto positivo, afecto negativo y síntomas somáticos. Trabajos más recientes señalan hallazgos similares con diferentes configuraciones. Así, Aguilera, Carreño y Juárez (2004), en un estudio realizado con una muestra de 310 escolares de zona rural, obtienen a partir del coeficiente alpha de Cronbach un índice de .76 y una estructura de 4 factores, aunque con distinta conformación de reactivos respecto del estudio de Radloff (1977). En el trabajo de González-Forteza et al., (2011), se aplicó a 57,403 estudiantes de nivel medio superior y superior de la ciudad de México, como parte de una cédula más amplia que recaba información sobre la salud física y mental, la familia y el entorno social de cada alumno. Los resultados indican alta consistencia interna $(\alpha=0.83)$ y la identificación de cuatro factores: afecto deprimido, afecto positivo, somatización e interpersonal. En su versión de 7 reactivos un trabajo reciente es el de Franco et al. (2018), que muestra invarianza factorial en los diferentes grupos de análisis, por lo que se sugiere su uso en encuestas, estudios tempranos y poblacionales. Estas diferencias en los resultados de estudios de valoración de las propiedades psicométricas del CES-D obtenidos denotan la importancia de las características particulares de la cultura y de los grupos etarios. 
Otra problemática psicosocial que afecta a la población adolescente es la ideación suicida, que hace referencia a cualquier comportamiento suicida no fatal, que incluye "ideas, planes, deseos y expectativas de cómo llevar a cabo el suicidio" (Osnaya, Pérez, \& Castillo, 2012, p. 288). La ideación suicida se asocia estrechamente al intento suicida, el cual presenta mayor riesgo de ocurrencia cuando existe un plan para ello (Sarkisian et al., 2019). Respecto a los instrumentos utilizados en los estudios científicos para evaluar la ideación suicida se encuentra la Escala de Ideación Suicida de Roberts (EIS) (Pineda-Roa, Martínez, Corredor, Herazo, \& Campo-Arias, 2018; Rosales-Pérez, Córdova-Osnaya, \& Cortés-Granados, 2015), que contiene un formato breve de 4 preguntas, mediante el que se explora el grado de intensidad de las ideas y planes suicidas de un individuo. El análisis de sus propiedades psicométricas realizado por Cubillas, Román, Valdez y Galaviz (2012) arrojó una estructura de un solo factor y una consistencia interna de 0.78 .

Estrechamente asociada a la ideación suicida, se encuentra la impulsividad, concepto que se manifiesta por falta de control en las reacciones o pensamientos inmediatos, que puede llevar a las personas a experimentar situaciones emocionalmente dolorosas, vergüenza o rechazo interpersonal. Uno de los instrumentos que evalúan este constructo es la Escala de Impulsividad (EI) de Climent et al., (1989), integrada en la Drug Risk Scale, adaptada por González-Forteza, Andrade y Jiménez (1997b), en un estudio aplicado a 423 adolescente de la ciudad de México, en el que se reportó una estructura de un solo factor y una consistencia interna de .75. Ambas Escalas han sido utilizadas en México, en estudios que exploran su relación a otras variables psicosociales (González-Forteza, Ramos, Caballero, \& Wagner, 2003; González-Forteza et al., 2015; García et al., 2018).

Existen diversos instrumentos que exploran correlatos psicológicos asociados a la ideación suicida, depresión e impulsividad en adolescentes como la Escala de Relación con el padre y con la madre [RELPA, RELMA], que evalúan el afecto, la comu-

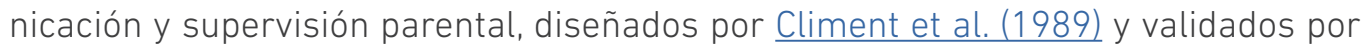
González-Forteza et al. (1999), a través de un estudio realizado con 816 estudiantes de 13 a 18 años, obteniendo un elevado nivel de confiabilidad ( $\alpha=0.90)$ y una estructura idéntica de tres factores; en el trabajo de Unikel y Gómez-Peresmitré (2004), la validación de ambos instrumentos, confirma igualmente una estructura de tres factores, con elevados niveles de confiabilidad $(\alpha=0.87$ a 0.94). Esta Escala ha sido utilizada para evaluar programas de prevención de riesgo suicida, junto con otras que miden variables como la depresión, ansiedad e ideación suicida (Córdova, Cubillas, \& Román, 2011). La Escala de Autoestima de Rosenberg (EAR), ampliamente utilizada en el ámbito internacional, como variable de análisis en programas de intervención (Díaz, Pérez, Sánchez, Moriana, \& Sánchez, 2018); en estudios realizados con población adolescente española se ha advertido que la EAR posibilita una evaluación global de la autoestima, con correlación moderada respecto de la ansiedad social, habilidades sociales y el pesimismo defensivo, y el análisis de regresión indicó que el comportamiento agresivo predice una autoestima baja (Caballo \& Salazar, 2018; Estévez et al., 2018; Ferradás, Freire, Regueiro, \& Valle, 2018). En un estudio en población bosnia femenina (Bratovcic, Mikic, Kostovski, Teskeredzic, \& Tanovic, 2015), la EAR mostró una estrecha correlación con la autopercepción; en población chilena (Silva et al., 2017) relacionó con sujetos con ideación suicida. En México, específicamente en Chiapas, el trabajo de Guitart, Rivas y Pérez (2011), que analizó la relación entre identidad étnica y autoestima en 517 estudiantes universitarios, confirmó la estructura unifactorial de la Escala y una consistencia interna satisfactoria. Ha sido 
Pág 74

Dada entonces la necesidad de probar su ajuste en la población en que se emplea, considerando los diversos sistemas sociales y culturales (Casullo, 2009), a partir del análisis de sus propiedades psicométricas, en el presente trabajo, se explora la validez y estructura factorial de las escalas de Ideación Suicida de Roberts, de Depresión de Centro de Estudios Epidemiológicos [CES-D], de Autoestima de Rosenberg, de Impulsividad, de Relación con Papá y Relación con Mamá de Climent et al. en adolescentes del estado de Chiapas en México. validada en diversos estudios, siendo uno de los más reconocidos el de Alessandri, Vecchione, Eisenberg y Laguna (2015), quienes corroboran la estructura robusta de dos factores de la Escala.

La falta de datos normativos actualizados en población adolescente de México de estos instrumentos de evaluación imposibilita su uso en estudios tanto de detección como de prevención de las problemáticas psicosociales con las que se relacionan. Dada entonces la necesidad de probar su ajuste en la población en que se emplea, considerando los diversos sistemas sociales y culturales (Casullo, 2009), a partir del análisis de sus propiedades psicométricas, en el presente trabajo, se explora la validez y estructura factorial de las escalas de Ideación Suicida de Roberts, de Depresión de Centro de Estudios Epidemiológicos [CES-D], de Autoestima de Rosenberg, de Impulsividad, de Relación con Papá y Relación con Mamá de Climent et al. en adolescentes del estado de Chiapas en México.

\section{Método}

El presente estudio tiene un diseño no experimental, de tipo transversal ex-post facto; mediante un muestreo no probabilístico, por conveniencia, se recolectó una muestra desde el 2011 hasta el 2015 de 4,759 adolescentes de tercer año de bachillerato, del estado de Chiapas, entidad ubicada en el sureste de México. En este lugar se presenta un alto grado de marginación y rezago social, ya que de acuerdo con la Secretaría de Desarrollo Social -SEDESOL- (2018), 77.08 \% de su población es pobre y $28.08 \%$ vive en pobreza extrema; ocupa el primer lugar en rezago educativo ${ }^{1}(29.0 \%$ de su población); además, $81.1 \%$ carece de seguridad social, $52.3 \%$ de servicios básicos de vivienda y $15 \%$ no tiene acceso a servicios de salud, condiciones que se exacerban en comunidades indígenas (Villafuerte-Solís, 2015). El trabajo se realizó en escuelas de la ciudad de Tuxtla Gutiérrez, capital del estado que acoge las sedes del gobierno estatal, congreso y servicios comerciales, así como en distintas localidades de la entidad que se dedican a actividades agrícolas y de servicios.

La distribución por sexo de los participantes fue de 3,275 mujeres (68.8\%) y 1,484 hombres (31.2\%), con un promedio de edad de 18.4 años y una desviación estándar de 1.67 años; de los cuales, $93.3 \%$ procede de centros escolares públicos y $6.7 \%$ privados, la mitad de ellos (50.5\%) de Tuxtla Gutiérrez, capital del estado, y el resto de diferentes localidades del interior del mismo; $14.7 \%$ trabajaba al momento de la recolección de información y $85.3 \%$ no.

\section{Técnicas e instrumentos de recolección de datos}

Se utilizaron instrumentos estandarizados en población mexicana, de formato breve, que miden presencia y severidad de cada variable de estudio. Antes de su aplicación se llevó a cabo un estudio piloto para probar su adecuación semántica y fácil respuesta.

Escala de Ideación Suicida de Roberts (EIS) (Roberts \& Chen, 1995). Consta de cuatro reactivos (tres de la escala de Roberts y uno de la CES-D: "No podía seguir adelante") referentes a pensamientos sobre la propia muerte y quitarse la vida, ocurridos durante los últimos siete días, en el mismo formato de respuestas que la CES-D: $1=0$ días, $2=1-2$ días, 3= 3-4 días y 4= 5-7 días, así que el rango teórico fluctúa de 4 a 16 . Ha sido evaluada satisfactoriamente en su consistencia interna con índices alpha de Cronbach $\geq .80$ y validez estructural de un factor (González-Forteza, Ramos, Mariño. \& Pérez, 2002; González-Forteza, Ramos, Vignau, \& Ramírez, 2001).

1. En México, el rezago educativo es definido como la condición en la que se encuentra una persona mayor de 15 años cuando no ha concluido su enseñanza básica. Por mandato constitucional, en este país, desde 1993 la enseñanza básica incluye los niveles de primaria y secundaria de primer ciclo (seis y tres años, respectivamente). (Núñez Barboza, 2005). 
Escala de Depresión del Centro de Estudios Epidemiológicos (CES-D, por sus siglas en inglés) (Radloff, 1977). Consta de 20 reactivos que evalúan la sintomatología depresiva durante la semana previa a su aplicación, con opciones de respuesta: $0=0$ días, 1= 1-2 días, $2=3-4$ días y $3=5-7$ días, por lo que el rango teórico fluctúa de 0 a 60. La consistencia interna $(\geq 0.83)$ y diversos tipos de validez han resultado consistentemente satisfactorios en estudios con población escolar adolescente mexicana rural (Aguilera et al., 2004) y urbana (González-Forteza et al., 2011).

Escala de Impulsividad (EI) (Climent et al., 1989). Consta de cinco reactivos y cuatro opciones de respuestas tipo Likert, que son: $1=$ casi nunca, $2=$ a veces, $3=$ con frecuencia y $4=$ con mucha frecuencia, para evaluar la frecuencia con que las personas refieren haber realizado acciones con escasa reflexión acerca de las consecuencias que puede implicar el llevarlas a cabo. Ha sido validada para población mexicana por González-Forteza, Andrade, \& Jiménez (1997b).

Escala de Autoestima de Rosenberg (EAR) (Rosenberg, 1965). Consta de 10 reactivos: 5 enunciados positivos y 5 negativos con opciones de respuesta: $1=$ Totalmente de acuerdo, 2= De acuerdo, 3= En desacuerdo, y 4= Totalmente en desacuerdo. Al recodificar los reactivos negativos, el rango teórico fluctúa de 10 a 40, de modo que a mayor puntaje más alta autoestima. En adolescentes mexicanos esta Escala ha obtenido índices de consistencia interna aceptables ( $\geq$.74) (Jiménez et al., 2007).

Escalas de Relación con el padre y con la madre (RELPA, RELMA) (Climent et al., 1989). Cada una consta de 15 reactivos con cuatro opciones de respuesta tipo Likert, que son: 1 = Casi nunca, $2=$ A veces, $3=$ Con frecuencia y $4=$ Con mucha frecuencia, por lo que el rango teórico de cada Escala es de 15 a 60; en la que a más puntaje, mejor calidad de la relación paterna/ materna percibida por el hijo(a). Estas Escalas han mostrado ser consistentemente confiables con coeficientes alpha de Cronbach $\geq .80$ y tres factores en cada una: afecto, comunicación y supervisión (González-Forteza et al., 1999).

\section{Procedimiento}

El proyecto fue avalado científica y éticamente por la Dirección de Investigación y Posgrado de la Universidad de Ciencias y Artes de Chiapas, México. Durante el proceso de inscripción de los estudiantes al centro escolar, se expuso a los padres de familia, los objetivos de la investigación y el uso confidencial y anónimo de la información recolectada, se obtuvo su consentimiento informado, así como el de los estudiantes, quienes aceptaron participar de forma voluntaria y autorizaron el uso de la información con fines de investigación.

Las Escalas se administraron por psicólogos capacitados, mediante procedimiento estandarizado de instrucciones el cual fue supervisado por un equipo de apoyo habilitado para tal propósito.

Mediante el SPSS, versión 21, se conformó el fichero e inició la ruta de análisis. En primer lugar, para validar la consistencia interna de cada Escala, se calculó el coeficiente alpha de Cronbach, tanto de manera general como en la modalidad "si se elimina un elemento". En segundo término, para determinar la validez de constructo de cada Escala, se realizó el análisis factorial mediante la factorización de ejes principales y rotación Varimax. Con los resultados, se evaluaron las saturaciones en cada factor para identificar el sentido de los componentes hallados, así como su consistencia interna. Posteriormente, para valorar la capacidad de cada instrumento e identificar 
entre puntajes altos y bajos, se utilizó el procedimiento de comparación de medias de Mann-Whitney para grupos contrastados. En la prueba de grupos contrastados se compararon los puntajes globales de cada Escala que se situaron por debajo del percentil 25 contra aquéllos situados por arriba del percentil 75. Por último, se obtuvieron puntajes de referencia para cada Escala mediante la suma simple del promedio de cada una de ellas más una desviación estándar. Este puntaje de referencia se obtuvo tanto de manera general, como por sexo.

\section{Resultados}

Escala de Ideación Suicida.

El valor alpha de Cronbach para la EIS de 4 ítems fue de .776. La prueba de esfericidad de Bartlett sobre la Escala dio como resultado un $\mathrm{p} \leq .000$, por lo que el análisis factorial es válido. Los 4 ítems integran un solo factor denominado ideación suicida, el cual explica el $60.99 \%$ de la varianza (Tabla 1). El valor de referencia de la Escala general, redondeado a valores enteros, es de un punto $(M=.16+$ una D.E.=.79). Al distinguir por sexo, el punto de corte es de 1, tanto para hombres $(M=0.14+$ una D.E. $=0.61)$, como para mujeres $(M=0.17$ + una D.E.= 0.87). La prueba de grupos contrastados no se realizó pues el primer y tercer cuartil coinciden en 0 puntos.

Tabla 1. Índice de confiabilidad y análisis factorial de la

Escala de Ideación Suicida

\begin{tabular}{lc}
\hline $\begin{array}{l}\text { Alpha de Cronbach: .776 } \\
\text { Varianza explicada: } \mathbf{6 0 . 9 9 \%}\end{array}$ & Factor \\
\hline Ítem & $\mathbf{1}$ \\
\hline 3. Sentía que mi familia estaría mejor, si yo estuviera muerto(a) & .812 \\
4. Pensé en matarme & .701 \\
2. Tenía pensamientos sobre la muerte & .664 \\
1. Sentía que no podía seguir adelante & .593 \\
\hline
\end{tabular}

Escala de Depresión del Centro de Estudios Epidemiológicos.

La confiabilidad para la Escala general fue de .747. Al calcular el alpha de Cronbach en la modalidad "si el reactivo fuera removido" se comprobó que no hay evidencia numérica para eliminar alguno de ellos, por lo que se trabajó con los 20 ítems en su totalidad. La prueba de esfericidad de Bartlett sobre los 20 ítems ( $p \leq .000)$ confirmó la validez del análisis factorial, el cual sugirió una estructura de 4 factores, con autovalores superiores a 1, que explican el $51.63 \%$ de la varianza. El primero de ellos denominado Afecto deprimido, el segundo Somatización, el tercero Afecto Positivo y el cuarto Relaciones interpersonales. El ítem 10: Tenía miedo, presentó saturaciones semejantes tanto para el factor uno como para el factor dos; sin embargo, se asoció al factor dos en virtud de que presenta mayor correspondencia de constructo con los ítems que conforman tal factor. Todos los factores, con excepción del 4, presentaron valores de confiabilidad moderados o altos (Tabla 2). La prueba de grupos contrastados mediante la comparación de promedios de Mann-Whitney, indica diferencias estadísticamente significativas entre los cuartiles $(z=-41.64, p \leq .000)$. El punto de corte es de 16 puntos $(M=10.26+$ una D.E. $=5.85)$. Por sexo, el punto de corte para ambos coincide en 16 puntos; sin embargo, los puntajes para los hombres ( $M=10.2$ + una D.E. $=5.45)$ son, en promedio, más bajos y homogéneos que los puntajes de las mujeres $(M=10.29+$ una D.E.= 6.02). 
Tabla 2. Índices de confiabilidad y análisis factorial de la Escala CES-D

\begin{tabular}{|c|c|c|c|c|}
\hline $\begin{array}{l}\text { Alpha de Cronbach: .747 } \\
\text { Varianza explicada: } 51.63 \%\end{array}$ & & Factor & & \\
\hline Ítem & 1 & 2 & 3 & 4 \\
\hline Afecto deprimido & $\alpha=.826$ & & & \\
\hline 18. Me sentía triste & .731 & & & \\
\hline 6. Me sentí deprimido (a) & .671 & & & \\
\hline 3. No podía quitarme la tristeza & .579 & & & \\
\hline 17. Lloraba a ratos & .533 & & & \\
\hline 14. Me sentía solo (a) & .427 & & & \\
\hline 10. Tenía miedo & .357 & & & \\
\hline Somatización & & $\alpha=.706$ & & \\
\hline $\begin{array}{l}\text { 1. Me molestaron muchas cosas que generalmente no me } \\
\text { molestan }\end{array}$ & & .520 & & \\
\hline $\begin{array}{l}\text { 5. Tenía problemas para poner atención a lo que estaba } \\
\text { haciendo }\end{array}$ & & .506 & & \\
\hline 11. Dormía sin descansar & & .483 & & \\
\hline 15. Sentía que la gente era poco amigable & & .447 & & \\
\hline 7. Sentí que todo lo que hacía me costó mucho trabajo & & .427 & & \\
\hline 2. No tenía hambre, ni apetito & & .399 & & \\
\hline 13. Platiqué menos de lo normal & & .397 & & \\
\hline Afecto positivo & & & $\alpha=.822$ & \\
\hline 16. Disfruté de la vida & & & .799 & \\
\hline 12. Estaba feliz & & & .796 & \\
\hline 8. Veía el futuro con esperanza & & & .660 & \\
\hline 4. Sentía que era tan bueno como los demás & & & .658 & \\
\hline Relaciones Interpersonales & & & & $\alpha=.556$ \\
\hline 9. Pensé que mi vida era un fracaso & & & & .488 \\
\hline 19. Sentía que no le caía bien a los demás & & & & .463 \\
\hline 20. No podía seguir adelante & & & & .456 \\
\hline
\end{tabular}

Escala de Impulsividad.

El valor de alpha de Cronbach para la El de 5 ítems fue de .653. La prueba de esfericidad de Bartlett sobre la Escala dio como resultado un $\mathrm{p} \leq .000$, por lo que el análisis factorial es pertinente. Los 5 ítems integran un solo factor denominado Impulsividad, el cual explica el 42.99\% de la varianza (Tabla 3). El valor de referencia de la Escala general, redondeando a valores enteros, es de 11 puntos $(M=8.21+$ una D.E. $=2.40)$. Al distinguir por sexo, el punto de corte para los hombres es de 11 puntos ( $M=8.68+$ una D.E.= 2.6), mientras que para las mujeres es de 10 puntos ( $M=8+$ una D.E.=2.27). La comparación de promedios de Mann-Whitney fue significativa ( $z=-44.23, p \leq .000$ ), lo que confirmó que la Escala tiene la capacidad para cribar entre puntajes altos y bajos. 
Tabla 3. Índice de confiabilidad y análisis factorial de la

Escala de Impulsividad

\begin{tabular}{lc}
\hline $\begin{array}{l}\text { Alpha de Cronbach: .653 } \\
\text { Varianza explicada: } \mathbf{4 2 . 9 9 \%}\end{array}$ & Factor \\
\hline Ítem & $\mathbf{1}$ \\
\hline 2. Haces cosas impulsivamente & .622 \\
3. Corres riesgos (peligros) & .592 \\
1. Haces cosas arriesgadas, sólo porque son emocionantes & .583 \\
4. Haces lo que te gusta, sin pensar en las consecuencias & .547 \\
5. Te desesperas fácilmente con la gente & .315 \\
\hline Factorización de ejes principales No hay solución rotada &
\end{tabular}

Escala de Autoestima de Rosenberg.

El análisis de confiabilidad interna de la EAR tuvo un valor de a=.798. Al obtener el alpha de Cronbach en la modalidad: "si el reactivo fuera removido" se determinó no eliminar a alguno de los ítems. Una vez que se comprobó que la prueba de esfericidad de Bartlett arrojó un resultado significativo ( $p \leq .000)$, se realizó el análisis por factorización de ejes principales y rotación Varimax. Los resultados de este análisis evidenciaron dos factores que en conjunto explican el $62.19 \%$ de la varianza. El primero de ellos quedó integrado por los 5 ítems en sentido directo (valoración global positiva o de aceptación), mientras que el segundo factor quedó conformado por los 5 ítems inversos (valoración global negativa o de rechazo), coincidente con la estructura obtenida por Alessandri et al. (2015).

Los ítems 5 y 8 presentan saturaciones bajas en el segundo factor $(<.300)$, sin embargo, la saturación de los mismos ítems en el factor uno es aún inferior. Por tanto, con el propósito de no perder la posibilidad de comparar resultados con los obtenidos en otros trabajos que utilicen el mismo instrumento, los ítems no fueron eliminados. El coeficiente alpha de Cronbach calculado para cada uno de los factores confirmó la consistencia interna de ambos (Tabla 4).

Tabla 4. Índices de confiabilidad y análisis factorial de la Escala de Autoestima de Rosenberg

\begin{tabular}{lcc}
\hline $\begin{array}{l}\text { Alpha de Cronbach: .798 } \\
\text { Varianza explicada: } \mathbf{6 2 . 1 9 \%}\end{array}$ & \multicolumn{1}{l}{ Factor } \\
\hline Ítem & $\mathbf{a = . 8 2 6}$ & $\mathbf{2}$ \\
\hline & .939 \\
\hline 6. Tengo una actitud positiva hacia mí mismo(a) & .929 \\
2. Siento que tengo buenas cualidades & .915 \\
4. Soy capaz de hacer las cosas tan bien, como los demás & .908 & \\
7. En general, estoy satisfecho(a) conmigo mismo(a) & .762 & $\mathbf{\alpha = . 8 2 6}$ \\
1. Siento que soy una persona que vale, al menos como los demás & .793 \\
\hline & & .539 \\
9. A veces me siento inútil & & .666 \\
3. En general me inclino a pensar que soy un fracaso & & .298 \\
10. A veces pienso que soy un(a) bueno(a) para nada & & \\
5. Siento que no tengo mucho de qué presumir de mí & & \\
8. Desearía poder tener más respeto por mí mismo(a) & & \\
\hline Solución rotada: Métodovarimax & &
\end{tabular}


La comparación de promedios de Mann-Whitney fue significativa ( $z=-44.23, p \leq .000$ ), lo que confirmó la capacidad del instrumento para diferenciar los puntajes mayores y menores. El punto de corte de la EAR en general es de 35 puntos $(M=28.95+$ una D.E.= 4.02). Al diferenciar por sexo, se encontró que el punto de corte para los hombres es de 34 puntos $(M=27.51+$ una $D . E .=6.4)$ y de 35 puntos para mujeres $(M=28.96+$ una D.E.= 6.36).

Escalas de Relación con Papá.

El valor del coeficiente alpha de Cronbach para la Escala general fue de .941. Al evaluar este coeficiente en la modalidad "si se elimina el elemento", no se encontró evidencia para eliminar alguno de los 15 ítems, por lo que se trabajó con la totalidad de ellos. La prueba de esfericidad de Bartlett resultó significativa ( $p \leq .000)$. El análisis factorial sugiere la conformación de 3 factores que, en conjunto, explican el $71.32 \%$ de la varianza. La saturación de los ítems en la solución rotada permitió identificar los factores: Afecto, Comunicación y Supervisión, los cuales coinciden con la estructura encontrada en otros estudios realizados con población de contextos semejantes a éste (Tabla 5). La comparación de promedios de Mann-Whitney al evaluar los grupos extremos fue significativa ( $z=-42.153, p \leq .000)$. El punto de corte fue de 54 puntos $(M=42.07+$ una $D . E .=11.40)$. Por sexo, los puntajes de referencia fueron de 52 puntos para hombres $(M=41.02$ + una D.E. $=11.2)$ y de 54 puntos para mujeres $(M=42.54$ + una D.E. $=11.47)$.

Tabla 5. Índices de confiabilidad y análisis factorial de la Escala Relación con Papá

\begin{tabular}{|c|c|c|c|}
\hline \multirow{2}{*}{$\begin{array}{l}\text { Alpha de Cronbach: } .941 \\
\text { Varianza explicada: } 71.32 \% \\
\text { Ítem }\end{array}$} & \multicolumn{3}{|c|}{ Factor } \\
\hline & 1 & 2 & 3 \\
\hline Afecto & $\alpha=.920$ & & \\
\hline 6. ¿Muestra que se preocupa por ti? & .754 & & \\
\hline 7. ¿Trata de darte lo que necesitas? & .715 & & \\
\hline 5. ¿Se muestra interesado en ayudarte? & .684 & & \\
\hline 8. ¿Es justo contigo? & .645 & & \\
\hline 1. ¿Te demuestra afecto? & .610 & & \\
\hline 9. ¿Te expresa el amor que siente por tí? & .601 & & \\
\hline Comunicación & & $\alpha=.883$ & \\
\hline 4. ¿Habla contigo de tus problemas? & & .748 & \\
\hline 3. ¿Habla contigo de tu vida (planes, amigos(as) juegos, etc. & & .683 & \\
\hline 14. ¿Habla contigo sobre tus dudas sexuales? & & .676 & \\
\hline 12. ¿Le gusta hablar contigo sobre las cosas que haces? & & .634 & \\
\hline 2. ¿Realiza alguna actividad agradable contigo? & & .613 & \\
\hline $\begin{array}{l}\text { 15. ¿Te dice que las relaciones sexuales deben aplazarse hasta } \\
\text { la edad adulta? }\end{array}$ & & .441 & \\
\hline Supervisión & & & $\alpha=.798$ \\
\hline 10. ¿Sabe a dónde vas cuando sales? & & & .801 \\
\hline 11. ¿Sabe con quién estás cuando sales? & & & .762 \\
\hline 15. ¿Te obliga siempre a llegar a casa a la hora fijada? & & & .446 \\
\hline
\end{tabular}




\begin{abstract}
Escalas de Relación con Mamá.
El valor del coeficiente alpha para la Escala general fue de .905. En la modalidad "si se elimina el elemento", no se encontró evidencia para eliminar alguno de los 15 ítems, por lo que se trabajó con la totalidad de ellos. La prueba de esfericidad de Bartlett resultó significativa ( $\mathrm{p} \leq .000$ ), por lo que se procedió a realizar el análisis factorial. Los resultados de este análisis sugirieron, al igual que con la escala de RELPA, la conformación de 3 factores que explican el $64.16 \%$ de la varianza, aunque bajo un arreglo distinto (Tabla 6 ).
\end{abstract}

Tabla 6. Índices de confiabilidad y análisis factorial de la Escala Relación con Mamá

\begin{tabular}{|c|c|c|c|}
\hline $\begin{array}{l}\text { Alpha de Cronbach: } .905 \\
\text { Varianza explicada: } 64.16 \%\end{array}$ & & Factor & \\
\hline Ítem & 1 & 2 & 3 \\
\hline Afecto & $\alpha=.914$ & & \\
\hline 5. ¿Se muestra interesado en ayudarte? & .761 & & \\
\hline 1. ¿Te demuestra afecto? & .724 & & \\
\hline 9. ¿Te expresa el amor que siente por ti? & .721 & & \\
\hline 6. ¿Muestra que se preocupa por ti? & .703 & & \\
\hline 3. ¿Habla contigo de tu vida (planes, amigos(as) juegos, etc. & .682 & & \\
\hline 4. ¿Habla contigo de tus problemas? & .663 & & \\
\hline 2. ¿Realiza alguna actividad agradable contigo? & .607 & & \\
\hline 8. ¿Es justo contigo? & .590 & & \\
\hline 12. ¿Le gusta hablar contigo sobre las cosas que haces? & .575 & & \\
\hline 7. ¿Trata de darte lo que necesitas? & .569 & & \\
\hline Comunicación & & $\alpha=.677$ & \\
\hline 14. ¿Habla contigo sobre tus dudas sexuales? & & .762 & \\
\hline $\begin{array}{l}\text { 15. ¿Te dice que las relaciones sexuales deben aplazarse hasta la } \\
\text { edad adulta? }\end{array}$ & & .644 & \\
\hline 13. ¿Te obliga siempre a llegar a casa a la hora fijada? & & .357 & \\
\hline Supervisión & & & $\alpha=.915$ \\
\hline 11. ¿Sabe con quién estás cuando sales? & & & .821 \\
\hline 10. ¿Sabe a dónde vas cuando sales? & & & .805 \\
\hline
\end{tabular}

La confiabilidad de los factores es moderada para el segundo factor y alta para el primero y el tercero. Al estar conformado únicamente con dos ítems, se sugiere utilizar el tercer factor exclusivamente como un indicador. La comparación de promedios de Mann-Whitney al evaluar los grupos extremos fue significativa ( $z=-43.000, p \leq .000)$, lo que valida la capacidad de la Escala para diferenciar entre puntajes bajos y altos. El punto de corte fue de 57 puntos ( $M=50.25+$ una D.E.= 8.23). Por sexo, los puntajes de referencia fueron de 57 puntos para hombres $(M=48.12+$ una D.E. $=8.25)$ y de 59 puntos para las mujeres $(M=51.22+$ una D.E. $=8.04)$. En todos los casos, los puntajes son más altos respecto a los de la Escala de Relación con Papá.

\title{
Discusión y conclusiones
}

Las Escalas aplicadas (de Ideación Suicida de Roberts, de Depresión del Centro de Estudios Epidemiológicos de Radloff, de Autoestima de Rosenberg, y de Impulsividad, Relación con Papá y Relación con Mamá, las tres de Climent, Aragón y Plutchick) 
El análisis factorial de la EIS, obtiene un solo factor, en el cual la carga de cada ítem es consistente, por lo que se confirma su utilidad como instrumento de tamizaje. La El obtiene igualmente un solo factor que integra los cinco reactivos, corroborándose su validez de constructo. mostraron niveles satisfactorios ( $a \geq .740$ ) o muy satisfactorios ( $a \geq .900)$ de confiabilidad a través del coeficiente alpha de Cronbach, excepto la El que obtuvo un a=.653. Las pruebas de esfericidad de Bartlett, con valores de $p$ menores a .05 para todas las Escalas, indicaron la factibilidad del análisis factorial. Los análisis factoriales exploratorios realizados mostraron arreglos de ítems semejantes, más no iguales a los reportados en otros estudios en los que se han aplicado estas escalas, confirmando la validez de los constructos teóricos en los que se basó su diseño.

El análisis factorial de la EIS, obtiene un solo factor, en el cual la carga de cada ítem es consistente, por lo que se confirma su utilidad como instrumento de tamizaje. La El obtiene igualmente un solo factor que integra los cinco reactivos, corroborándose su validez de constructo.

El análisis factorial de la Escala CES-D, sugiere una estructura de cuatro factores: Afecto deprimido, Somatización, Afecto positivo y Relaciones interpersonales, bastante similar a la configuración reportada por Radloff (1977), excepto por los reactivos: Pensé que mi vida era un fracaso, el cual se dispone en el factor Relaciones interpersonales, no en el de Afecto deprimido en el que estaba originalmente; y Sentía que la gente era poco amigable, se ubica en el factor Somatización, mientras que en el trabajo de Radloff, se sitúa en el de Relaciones Interpersonales. También resulta similar a la estructura factorial obtenida por González-Forteza et al. (2011), excepto por los reactivos: Pensé que mi vida era un fracaso y No podía seguir adelante, que se ubican en el factor de Relaciones interpersonales, mientras que en el de González-Forteza et al. (2011) se sitúa en el de Afecto deprimido; y, el reactivo: Sentí que todo lo que hacía me costó mucho trabajo ubicado en el de Somatización y que en el estudio de González-Forteza et al., (2011) correspondía al factor Afecto positivo. Al respecto, Tatar, Kayiran, Saltukoglu, Zeybek-Ozkut y Emeksiz (2013), explican que la Escala no tiene una estructura de factores generalizada y estos reactivos se intercambian en las subescalas de afecto deprimido, somatización o relaciones interpersonales; lo que a su vez refiere la compleja y cambiante dinámica evolutiva del constructo (Villalobos \& Ortiz, 2012).

En la El, el reactivo Te desesperas fácilmente con la gente obtiene un bajo peso factorial, por lo que debe tenerse reservas de su uso para evaluar el constructo; asimismo, decrementa el nivel de confiabilidad de la prueba. Ello es similar al resultado obtenido por González-Forteza et al. (1997b) en el cual este reactivo obtiene también el menor peso factorial. La falta de otros estudios limita un análisis más profundo de este aspecto.

En la EAR, se integran dos factores, los pesos factoriales de los ítems positivos constituyen el primero de ellos, denominado como de auto mejoramiento (valoración positiva) y, el segundo, llamado de auto derogación (valoración negativa) (Góngora \& Casullo, 2009). El bajo peso factorial de dos de los reactivos: Siento que no tengo mucho de qué presumir de mí; y: Desearía poder tener más respeto por mí mismo, coincidente con lo referido en el trabajo de Schmitt y Allik (2005), hace necesario considerar su pertinencia dada la poca evidencia de su correspondencia con el constructo que se desea medir. En el estudio de Guitart et al. (2011), llevado a cabo con jóvenes universitarios de Chiapas, estos mismos reactivos alcanzan los puntajes de media más bajos, por lo que en ese como en este trabajo debe considerarse su conveniencia para su incorporación al constructo, en correspondencia con los valores y cultura de los participantes (Villalobos \& Ortiz, 2012). 
Por su parte, en la escala RELPA, se obtiene una estructura de tres factores: Afecto, Comunicación y Supervisión, coincidente con lo referido por González-Forteza et al. (1999), incluso en la configuración de los reactivos, excepto por el de: Realiza alguna actividad agradable contigo, situado en el factor Comunicación y no en el de Afecto. Estos tres factores sugieren que la relación con los padres tiene un componente afectivo que se manifiesta en la atención percibida por los jóvenes, una valoración del canal de comunicación que se establece en la relación padre-hijo(a) y el control parental que regula dicha relación. En el presente estudio la Escala RELMA, presenta una configuración distinta de reactivos, respecto al trabajo de González-Forteza et al. (1999), se agregan al factor de Afecto, los reactivos: ¿Habla contigo de tu vida (planea, amigos(as), juegos, etc.?; ¿Habla contigo de tus problemas?; y el de: ¿Le gusta hablar contigo sobre las cosas que haces?, del factor Comunicación; mientras que en este mismo factor, se incluye el reactivo: ¿Te obliga siempre a llegar a casa a la hora fijada?, del factor Supervisión. La carga factorial de los ítems indica que los factores de Afecto y Comunicación se asocian en un único factor conformado por los reactivos que miden ambas situaciones de la relación madre-hijo(a). El segundo factor, Comunicación, agrupa ítems vinculados con la vida sexual de los jóvenes, mientras que el tercer factor, al igual que en los padres, agrupa un par de reactivos asociados con la supervisión.

La composición de los reactivos que conforman la estructura factorial de la Escala CES-D y las Escalas RELPA y RELMA es distinta respecto de otros estudios, debido probablemente a las diferencias culturales y evolutivas en la expresión de la sintomatología depresiva (Heo, Choi, Yu, \& Nam, 2018; McCabe, Vermeesch, Hall, Peragallo, \& Mitrani, 2011; Villalobos, \& Ortiz, 2012), así como por la relación más íntima que el adolescente establece con la madre en familias tradicionales como las del estado de Chiapas (Gutiérrez Narváez, 2014), en las que se constituyen como núcleo integrador de las relaciones sociales e interpersonales que median la comunicación y afecto, y del padre como puntal del orden y la vida en sociedad.

La capacidad de cada instrumento analizado en el presente estudio para identificar puntajes altos y bajos, obtenida mediante la comparación de promedios para casos extremos, mostró diferencias estadísticamente significativas entre los grupos que definen los cuartiles 1 y 3 de todos los instrumentos, excepto para la EIS, pues el primero y tercer cuartil coinciden en 0 puntos. Ello indica su utilidad como instrumentos de tamizaje de fácil aplicación para distinguir significativamente entre los grupos de mayores y menores puntajes en cada Escala.

Los puntos de corte (PC) referidos para cada instrumento son los siguientes: Para la Escala CES-D, es de 16, mayor en mujeres (16) que en hombres (15); similar al obtenido por Veytia, González, Andrade y Oudhof (2012) y mayor al de González-Forteza et al. (2011), en una muestra con edad promedio de 17 años ( $P C=10)$, lo que abona al argumento de que la sintomatología depresiva aumenta con la edad Villalobos \& Ortiz, 2012); en la EIS, el PC=1, tanto en hombres como en mujeres, es igual a lo señalado en el estudio de González-Forteza et al. (2003); en la El el PC=11, mayor en hombres (11) que en mujeres (10), similar a lo referido por González-Forteza et al. (1997a); en la EAR, el PC= 35, mayor en mujeres (35) que en hombres (34); en la escala RELPA, el PC=54, mayor en mujeres (54) que en hombres (52), mientras que para la escala RELMA, el $P C=57$, mayor en mujeres (59), que en hombres (57). 
Los resultados confirman la alta confiabilidad (excepto para la El) y validez de constructo de las distintas Escalas de evaluación de aspectos psicosociales estudiadas en el presente estudio, lo que corrobora las adecuadas propiedades psicométricas de los instrumentos para su uso en adolescentes de México, hombres y mujeres de 18 años; no obstante, debe tomarse con reserva la incorporación de dos de los reactivos de la EAR, ya que presentan un bajo peso factorial, lo mismo que uno de los reactivos de la El. Con estas consideraciones, su formato y breve extensión permite su rápida aplicación en grupos de adolescentes de contextos afines, y la detección oportuna de casos de riesgo en términos de su presencia y severidad.

En virtud de que el presente estudio se llevó a cabo con adolescentes escolarizados, cuyas problemáticas pueden ser más específicas, sería de sumo interés profundizar en la indagación de las propiedades de los instrumentos evaluados en adolescentes no escolarizados y en sujetos de otros grupos de edad, para probar si existen o no diferencias entre distintos grupos etarios; además, deberán continuarse estudios con diseños más robustos que corroboren los resultados obtenidos y probar la funcionalidad de los instrumentos, en el conocimiento de que los resultados pueden variar en relación con la cultura y escolaridad de los sujetos.

\section{Referencias}

Aguilera, R. M., Carreño, M. S., \& Juárez, F. (2004). Características psicométricas de la CES-D en una muestra de adolescentes rurales mexicanos de zonas con alta tradición migratoria. Salud Mental, 27(6), 57-66. Recuperado de: https://www. redalyc.org/pdf/582/58262708.pdf

Alessandri, G., Vecchione, M., Eisenberg, N., \& Laguna, M. (2015). On the factor structure of the Rosenberg (1965) General Self-Esteem Scale. Psychological Assessment, 27(2), 621-635. doi: http://dx.doi.org/10.1037/pas0000073

Alexandrowicz, R.W., Jahn, R., \& Wancata, J. (2018). Assessing the dimensionality of the CES-D using multi-dimensional multi-level Rasch models. PLOS ONE, 13(5), 1-19, e0197908. doi: https://doi.org/10.1371/journal.pone.0197908

Auerbach, R. P., Stewart, J. G., \& Johnson, S. L. (2017). Impulsivity and suicidality in adolescent inpatients. J. Abnorm Child Psychol, 45, 91-103. doi: https://doi. org/10.1007/s10802-016-0146-8

Baader M., T., Molina F., J. L., Venezian B., S., Rojas C., C., Farías S., R., Fierro-Freixenet, C., Backenstrass, M., \& Mundt, Ch. (2012). Validación y utilidad de la encuesta PHQ-9 (Patient Health Questionnaire) en el diagnóstico de depresión en pacientes usuarios de atención primaria en Chile. Revista Chilena de Neuro-psiquiatría, 50(1), 10-22. doi: http://dx.doi.org/10.4067/S0717-92272012000100002

Baron, E. C., Davies, T., \& Lund, C. (2017). Validation of the 10-item Centre for Epidemiological Studies Depression Scale (CES-D-10) in Zulu, Xhosa and Afrikaans populations in South Africa. BMC Psychiatry, 17, 6 doi: https://doi.10.1186/ s12888-016-1178-x

Bi, B., Liu, W., Zhou, D., Fu, X., Qin, X., \& Wu, J. (2017). Personality traits and suicide attempts with and without psychiatric disorders: analysis of impulsivity and neuroticism. BMC Psychiatry, 17(294), 1-9. doi: https://doi10.1186/s12888-017-1453-5

Borges, G., Orozco, R., Villatoro, J., Medina-Mora, M. E., Fleiz, C., \& Díaz-Salazar, J. (2019). Suicide ideation and behavior in Mexico: Encodat 2016. Salud Publica Mex., 61, 6-15. doi: https://doi.org/10.21149/9351

Bratovcic, V., Mikic, B., Kostovski, Z., Teskeredzic, A., \& Tanovic, I. (2015). Relations between different dimensions of self-perception, self-esteem and body mass index of female students. Int. J. Morphol., 33(4), 1338-1342. Recuperado de: https://scielo.conicyt.cl/pdf/ijmorphol/v33n4/art24.pdf 
Caballero Domínguez, C.C., Suárez Colorado, Y.P., \& Bruges Carbonó, H.D. (2015). Características de inteligencia emocional en un grupo de universitarios con y sin ideación suicida. Revista CES Psicología, 8(2), 138-155. Recuperado de: http://revistas.ces.edu.co/index.php/psicologia/article/view/3211/2426

Caballo, V. E. \& Salazar, I. C. (2018). La autoestima y su relación con la ansiedad social y Las habilidades sociales1 Behavioral Psychology / Psicología Conductual, 26(1), 23-53. Recuperado de: https://behavioralpsycho.com/wp-content/ uploads/2018/09/02.Caballo 26-1a.pdf

Casullo, Ma. M. (2009). La evaluación psicológica: modelos, técnicas y contextos. Revista Iberoamericana de Diagnóstico y Evaluación Psicológica, 27(1), 9-28. Recuperado de: https://www.redalyc.org/pdf/4596/459645443002.pdf

Climent, C. E., Aragón, L. V., \& Plutchick, R. (1989). Predicción del riesgo de uso de drogas por parte de estudiantes de secundaria. Bol of Sanit Panam, 107(6), 568576. Recuperado de: http://hist.library.paho.org/Spanish/BOL/v107n6p568.pdf

Córdova Moreno, M. A., Cubillas Rodríguez, M. J., \& Román Pérez, R. (2011). ¿Es posible prevenir el suicidio? Evaluación de un programa de prevención en estudiantes de bachillerato. Pensamiento Psicológico, 9(17), 21-32. Recuperado de: http://www. scielo.org.co/scielo.php?script=sci arttext\&pid=S1657-89612011000200003\&lng=en\&tlng=es

Courtin, E., Knapp, M., Grundy, E., \& Avendano-Pabon, M. (2015). Are different measures of depressive symptoms in old age comparable? An analysis of the CES-D and Euro-D scales in 13 countries. Int. J. Methods Psychiatr. Res., 24(4), 287-304. Recuperado de: https://onlinelibrary.wiley.com/doi/epdf/10.1002/mpr.1489

Cubillas Rodríguez, M. J., Román Pérez, R., Valdez, E. A., \& Galaviz Barreras, A. L. (2012). Depresión y comportamiento suicida en estudiantes de educación media superior en Sonora. Salud Mental, 35, 45-50. Recuperado de: http://www.scielo. org. $\mathrm{mx} / \mathrm{pdf} / \mathrm{sm} / \mathrm{v} 35 \mathrm{n} 1 / \mathrm{v} 35 \mathrm{n} 1 \mathrm{a} 7 . \mathrm{pdf}$

Díaz-González, M. C., Pérez Dueñas, C., Sánchez-Raya, A., Moriana Elvira, J. A., \& Sánchez Vázquez, V. (2018). Mindfulness-based stress reduction in adolescents with mental disorders: A randomised clinical trial. Psicothema, 30(2), 165-170. doi: https://doi.10.7334/psicothema2017.259

Estévez, E., Jiménez, T. I., \& Moreno, D. (2018). Aggressive behavior in adolescence as a predictor of personal, family, and school adjustment problems. Psicothema, 30(1), 66-73. doi: https://doi.10.7334/psicothema2016.294

Ferradás Canedo, M. M., Freire Rodríguez, C., Regueiro Fernández, B., \& Valle Arias, A. (2018). Defensive pessimism, self-esteem and achievement goals: A person-centered approach. Psicothema, 30(1), 53-58. doi: https://doi.10.7334/psicothema2017.199

Franco-Díaz, K. L., Fernández-Niño, J. A., \& Astudillo-García, C. I. (2018). Prevalencia de síntomas depresivos e invarianza factorial de la Escala de Depresión del Centro de Estudios Epidemiológicos (CES-D) en población indígena mexicana. Biomédica, 38, 127-140. doi: https://doi.org/10.7705/biomedica.v38i0.3681

García Lara, G. A., Ocaña Zúñiga, J., Cruz Pérez, O., Hernández Solís, S., Pérez Jiménez, C. E., \& Cabrera Méndez, M. (2018). Predictors of suicidal ideation and depressive symptoms among adolescents in Chiapas, Mexico. Ciência \& Saúde Coletiva, 23(4), 1089-1096. doi: https://doi.10.1590/1413-81232018234.14492016

García Lara, G. A., Ocaña Zúñiga, J., Hernández Solís, S., \& Cruz Pérez, O. (s/a). Iniciación sexual y uso de preservativos en adolescentes de zonas rurales indígenas y urbanas de Chiapas. En prensa.

Góngora, V. N., \& Casullo, Ma. M. (2009). Validación de la escala de autoestima de Rosenberg en población general y en población clínica de la Ciudad de Buenos 
Aires. Revista Iberoamericana de Diagnóstico y Evaluación Psicológica, 27(1), 179194. Recuperado de: https://www.redalyc.org/pdf/4596/459645443010.pdf

González, C., Hermosillo, A., Vacio-Muro, M. A., Peralta, R., \& Wagner, F. (2015). Depresión en adolescentes. Un problema oculto para la salud pública y la práctica clínica. Bol Med Hosp Infant Mex, 72(2), 149-155. Recuperado de: http://www.scielo.org.mx/pdf/bmim/v72n2/1665-1146-bmim-72-02-00149.pdf

González-Forteza, C., Andrade, P., \& Jiménez, J. A. (1997a). Estresores cotidianos familiares, sintomatología depresiva e ideación suicida en adolescentes mexicanos. Acta Psiquiátrica Psicológica América Latina, 43(4), 319 - 326.

González-Forteza, C., Andrade, P., \& Jiménez, J. A. (1997b). Recursos psicológicos relacionados con el estrés cotidiano en una muestra de adolescentes mexicanos. Salud Mental, 20(1), 27-35. Recuperado de: http://www.revistasaludmental.mx/index.php/ salud mental/article/view/639/638

González-Forteza, C., Jiménez Tapia, A., Pérez Campuzano, E., Ramos Lira, L., Caballero Gutiérrez, M. A., \& Saltijeral Méndez, M. T. (1999). Padres afectivos: apoyo para la autoestima de sus hijos adolescentes. Psicología Conductual, 7(3), 501-507. Recuperado de: https://www.scopus.com/record/display.uri?eid=2-s2.0-0033403627\&origin=inward\&txGid=ef8da1 dbff62b1f16a6b0838b80f516d

González-Forteza, C., Juárez López, C. E., Montejo León, L. de los A., Oseguera Díaz, G., Wagner Echeagaray, F. A., \& Jiménez Tapia, A. (2015). Ideación suicida y su asociación con drogas, depresión e impulsividad en una muestra representativa de estudiantes de secundaria del estado de Campeche, México. Acta Universitaria, 25(NE-2), 29-34. doi: https://doi.10.15174/au.2015.862

González-Forteza, C., Ramos, L., Mariño, M. C., \& Pérez, E. (2002). Vidas en riesgo: conducta suicida en adolescentes mexicanos. Acta Psiq Psic Am Lat, 48(1-4), 74-84.

González-Forteza, C., Ramos, L., Caballero, M. Á., \& Wagner, F. A. (2003). Correlatos psicosociales de depresión, ideación e intento suicida en adolescentes mexicanos. Psicothema, 15(4), 524 - 532. Recuperado de: http://www.psicothema. com/pdf/1102.pdf

González-Forteza, C., Ramos Lira, L., Vignau Brambila, L. E., \& Ramírez Villarreal, C. (2001). Abuso sexual e intento suicida: Asociación con el malestar depresivo y la ideación suicida actuales en adolescentes. Salud Mental, 24(6), 16-25. Recuperado de: https://www.redalyc.org/pdf/582/58262403.pdf

González-Forteza, C., Solís, C., Jiménez, A., Hernández, I., González, A., Juárez, F., Medina-Mora, M. E., \& Fernández-Varela, H. (2011). Confiabilidad y validez de la escala de depresión CES-D en un censo de estudiantes de nivel medio superior y superior en la Ciudad de México. Salud Mental, 34, 53-59. Recuperado de: https://www.medigraphic.com/pdfs/salmen/sam-2011/sam111g.pdf

Gray, J. S., Brionez, J., Petros, T., \& Gonzaga, K. T. (2018). psychometric evaluation of depression measures with Northern Plains Indians. American Journal of Orthopsychiatry. Advance online publication. doi: http://dx.doi.org/10.1037/ ort0000309

Guitart, E. M., Rivas Damián, M. J., \& Pérez Daniel, M. R. (2011). Identidad étnica y autoestima en jóvenes indígenas y mestizos de San Cristóbal de las Casas (Chiapas, México). Acta Colombiana de Psicología, 14(1), 99-108. Recuperado de: http://www.scielo.org.co/pdf/acp/v14n1/v14n1a09.pdf

Gutiérrez J.P., Rivera-Dommarco, J., Shamah-Levy, T., Villalpando-Hernández, S., Franco, A., Cuevas-Nasu L., ... Hernández-Ávila M. (2012). Encuesta Nacional de Salud y Nutrición 2012. Resultados nacionales. Cuernavaca, México: Instituto Nacional de Salud Pública. Recuperado de: https://ensanut.insp.mx/encuestas/ensanut2012/doctos/informes/ENSANUT2012ResultadosNacionales.pdf 
Gutiérrez Narváez, M. J. (2014). Identidad, familia y racismo en San Cristóbal de las Casas. San Cristóbal de las Casas, Chiapas, México: UNICACH / CESMECA.

Heo, E. H., Choi, K. S., Yu, J. C., \& Nam, J. A. (2018) Validation of the center for epidemiological studies depression scale among Korean adolescents. Psychiatry Investig, 15(2), 124-132. doi: https://doi.org/10.30773/pi.2017.07.19

Horowitz, L.M., Bridge J. A., Teach, S.J., Ballard, E., Klima, J., Rosenstein, D.L., ... Pao M. (2012). Ask Suicide-Screening Questions (ASQ). A Brief Instrument for the Pediatric Emergency Department. Archives of Pediatrics and Adolescent Medicine, 166(12), 1170-1176. Recuperado de: https://jamanetwork.com/journals/jamapediatrics/fullarticle/1363508

Instituto Nacional de Psiquiatría Ramón de la Fuente Muñiz [INPRFM]. (2017a). Encuesta Nacional de consumo de drogas, alcohol y tabaco, 2016-2017. Reporte de alcohol. Ciudad de México, México: INPRFM. Recuperado de: https://drive.google. com/file/d/1rMLKaWy34GR51sEnBK2-u2q BDK9LA0e/view

Instituto Nacional de Psiquiatría Ramón de la Fuente Muñiz [INPRFM]. (2017b). Encuesta Nacional de consumo de drogas, alcohol y tabaco, 2016-2017. Reporte de drogas. Ciudad de México, México: INPRFM. Recuperado de: https://drive.google. com/file/d/1zIPBiYB3625GBGIW5BX0TT YQN73eWhR/view

Harry, M., \& Crea, Th. M. (2018). Examining the measurement invariance of a modified CES-D for American Indian and non-Hispanic White adolescents and young adults. Psychological Assessment, 30(8), 1107-1120. doi: https://doi.org/10.1037/ pas0000553

Jiménez, A., Mondragón, L., \& González-Forteza, C. (2007). Self-esteem, depressive symptoms, and suicidal ideation in adolescents: Results of three studies. Salud Mental, 30(5), 20-26. Recuperado de: https://www.medigraphic.com/pdfs/salmen/sam-2007/sam075d.pdf

Mariño, M.C., Medina-Mora, M.E., Chaparro, J., \& González-Forteza, C. (1993). Confiabilidad y estructura factorial del CES-D en una muestra de adolescentes mexicanos. Rev Mex Psicol, 10(2), 141-145.

McCabe, B. E., Vermeesch, A. L., Hall, R. F., Peragallo, N. P., \& Mitrani, V. B. (2011). Acculturation and the Center for Epidemiological Studies-Depression Scale for Hispanic women. Nursing Research, 60(4), 270-275. doi: http://dx.doi. org/10.1097/NNR.0b013e318221b8dc

Miedema, S. S., Haardörfer, R., \& Yount, K. M. (2018). Comparable or not? Assessing measurement invariance of a modified Center for Epidemiologic Studies-Depression Scale between sexual minority and nonsexual minority men in five Asia-Pacific countries. Psychology of Men \& Masculinity. Advance online publication. doi: http://dx.doi.org/10.1037/men0000172

Núñez Barboza, M. (2005). El rezago educativo en México: dimensiones de un enemigo silencioso y modelo propuesto para entender las causas de su propagación. Revista Interamericana de Educación de Adultos, 27(2), 29-70. Recuperado de: https://www.redalyc.org/pdf/4575/457545128002.pdf

Organización Panamericana de la Salud. (2014). Prevención del suicidio: un imperativo global. Washington, DC: OPS. Recuperado de: https://apps.who.int/iris/bitstream/handle/10665/136083/9789275318508 spa.pdf;jsessionid=DF584C30BE328184ABAC4COCFBBC82DA? sequence $=1$

Osnaya, M. C., Pérez, J. C. R., \& Castillo, M. B. G. (2012). ideación suicida y variables asociadas en jóvenes universitarios del estado de Hidalgo (México): Comparación por género. Psicogente, 15(28), 287-301. Recuperado de: http://www.redalyc.org/pdf/4975/497552361006.pdf

Palacios, D. J. (2015). Estimación psicométrica de la escala de autoeficacia ante conductas de riesgo para adolescentes en México. Psychosocial Intervention, 1, 1-7. 
Recuperado de: https://reader.elsevier.com/reader/sd/pii/S113205591500006X?token=B9498692D117DB808B29712A28EFEA121FCAA6DBFCE85323FB4BA100AB28BBAB20A5129358804FCB3AF638164D1FCA05

Pineda-Roa, C.A., Martínez, Á.P., Corredor, D.Y., Herazo, E., \& Campo-Arias, A. (2018). Hallazgos psicométricos de la escala para ideación suicida del centro de estudios epidemiológicos en adolescentes escolarizados de Samacá, Boyacá, Colombia. Revista Biosalud, 17(2), 47-55. doi: https://doi.10.17151/biosa.2018.17.2.4

Radloff, L. (1977). The CES-D scale: A self-report depression scale for research in the general population. Applied Psychol Meas, 1, 385-401. doi: http://doi. org/10.1177/014662167700100306

Rosales-Pérez, J. C., Córdova-Osnaya, M., \& Cortés-Granados, R. (2015). Confiabilidad y validez de la Escala de Ideación Suicida de Roberts. Journal of Behavior, Health \& Social Issues, 7(2), 31-41. doi: https://doi.10.5460/jbhsi.v7.2.44302

Rosenberg, M. (1965). Society and the adolescent self-image. Princeton, N.J: Princeton: University Press.

Roberts, E., \& Chen, Y. W. (1995). Depressive symptoms and suicidal ideation among Mexican origin and Anglo adolescents. J. Am Acad Child Adolesc Psychiatry, 34(1), 81-90. doi: http://doi.org/10.1097/00004583-199501000-00018

Sánchez, J. C., Villarreal, M. E., \& Musitu, G. (2013). Capítulo 12. Ideación suicida. En G. Musitu Ochoa (Coord.). Adolescencia y familia: nuevos retos en el siglo XXI. (pp. 273-290). México: Trillas.

Sarkisian, K. L., Van Hulle, C. A., \& Hill Goldsmith, H. (2019). Brooding, inattention, and impulsivity as predictors of adolescent suicidal ideation. Journal of Abnormal Child Psychology, 47(2), 333-344. doi: https://doi.org/10.1007/s10802-0180435-5

Schmitt, Dp., \& Allik, J. (2005). Simultaneous administration of the Rosenberg Self-Esteem Scale in 53 nations: exploring the universal and culture-specific features of global self-esteem. J. Pers Soc Psychol, 89, 623-42. doi: https://dx.doi. org/10.1037/0022-3514.89.4.623

Secretaría de Desarrollo Social [SEDESOL]. (2018). Informe anual sobre la situación de pobreza y rezago social 2018. Subsecretaría de Planeación, Evaluación y Desarrollo Regional. Chiapas. Recuperado de: https://www.gob.mx/cms/uploads/ attachment/file/288980/Chiapas.pdf

Silva, D., Valdivia, M., Vicente, B., Arévalo, E., Dapelo, R., \& Soto, C. (2017). Intento de suicidio y factores de riesgo en una muestra de adolescentes escolarizados de Chile. Revista de Psicopatología y Psicología Clínica, 22, 33-42. doi: https:// doi.10.5944/rppc.vol.22.num.1.2017.16170

Tatar, A., Kayiran, S. M., Saltukoglu, G., Zeybek Ozkut, E. S., \& Emeksiz, M. (2018). Analysis of the Center for Epidemiologic Studies Depression Scale (CES-D) in Children and Adolescents from the Perspective of the Item Response Theory. Klinik Psikofarmakoloji Bülteni / Bulletin of Clinical Psychopharmacology, 23(3), 242-253. doi: http://dx. doi.org/10.5455/bcp.20130324030600

Tomitaka, S., Kawasaki, Y., Ide, K., Akutagawa, M., Ono, Y., \& Furukawa, T. A. (2018). Distribution of item responses and total item scores for the Center for Epidemiologic Studies Depression Scale (CES-D): Data from the Irish Longitudinal Study on Ageing (TILDA). PLoS ONE, 13(8), e0202607. doi: https://doi.org/10.1371/journal. pone.0202607

Unikel, C., \& Gómez-Peresmitré, G. (2004). Validez de constructo de un instrumento para la detección de factores de riesgo en los trastornos de la conducta alimentaria en mujeres mexicanas. Salud Mental, Febrero, 27(1), 39-49. Recuperado de: https://www.medigraphic.com/pdfs/salmen/sam-2004/sam041e.pdf 
Veytia López, M., González Arratia, López Fuentes, N. I., Andrade Palos, P., \& Oudhof, H. (2012). Depresión en adolescentes: El papel de los sucesos vitales estresantes. Salud Mental, 35, 37-43. Recuperado de: https://www.redalyc.org/pdf/582/58223290006.pdf

Villafuerte-Solís, D. (2015). Crisis rural, pobreza y hambre en Chiapas. LiminaR, 13(1), 13-28. Recuperado de: http://www.scielo.org.mx/scielo.php?script=sci arttex-

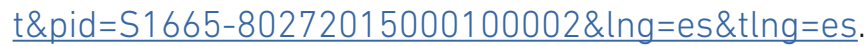

Villalobos-Galvis, F. H., \& Ortiz-Delgado, L.A. (2012). Características psicométricas de la escala CES-D en adolescentes de San Juan de Pasto (Colombia). Avances en Psicología Latinoamericana, 30(2), 328-340. Recuperado de: https://revistas. urosario.edu.co/index.php/apl/article/view/1477/1965

Yang, W., Xiong, G., Garrido, L. E., Zhang, J. X., Wang, M. C., \& Wang, C. (2018). Factor structure and criterion validity across the full scale and ten short forms of the CES-D among Chinese adolescents. Psychological Assessment, 30(9), 1186-1198. doi: $\underline{\text { http://dx.doi.org/10.1037/pas0000559 }}$ 